\title{
UAV-BASED 3D VIRTUAL TOUR CREATION
}

\author{
U. G. Sefercik ${ }^{1, *}$, T. Kavzoglu ${ }^{1}$, M. Nazar ${ }^{1}$, C. Atalay ${ }^{2}$, M. Madak ${ }^{3}$ \\ ${ }^{1}$ Dept. of Geomatics Engineering, Gebze Technical University, Kocaeli, Turkey - (sefercik, kavzoglu, mnazar)@gtu.edu.tr \\ ${ }^{2}$ Dept. of Geomatics Engineering, Bulent Ecevit University, Zonguldak, Turkey - canatalay@ beun.edu.tr \\ ${ }^{3}$ Dept. of Digitalization, Gizil Energy Inc., Istanbul, Turkey - muhammedmadak@ gizilenerji.com.tr
}

KEY WORDS: UAV, SFM, 3D Textured Mesh Model, Virtual Reality, Unity

\begin{abstract}
:
Lately, improvements in game engines have increased the interest in virtual reality (VR) technologies, that engages users with an artificial environment, and have led to the adoption of VR systems to display geospatial data. Because of the ongoing COVID-19 pandemic, and thus the necessity to stay at home, VR tours became very popular. In this paper, we tried to create a three-dimensional (3D) virtual tour for Gebze Technical University (GTU) Southern Campus by transferring high-resolution unmanned air vehicle (UAV) data into a virtual domain. UAV data is preferred in various applications because of its high spatial resolution, low cost and fast processing time. In this application, the study area was captured from different modes and altitudes of UAV flights with a minimum ground sampling distance (GSD) of $2.18 \mathrm{~cm}$ using a $20 \mathrm{MP}$ digital camera. The UAV data was processed in Structure from Motion (SfM) based photogrammetric evaluation software Agisoft Metashape and high-quality 3D textured mesh models were generated. Image orientation was completed using an optimal number of ground control points (GCPs), and the geometric accuracy was calculated as $\pm 8 \mathrm{~mm}$ ( $\sim 0.4$ pixels). To create the VR tour, UAV-based mesh models were transferred into the Unity game engine and optimization processes were carried out by applying occlusion culling and space subdivision algorithms. To improve the visualization, 3D object models such as trees, lighting poles and arbours were positioned on VR. Finally, textual metadata about buildings and a player with a first-person camera were added for an informative VR experience.
\end{abstract}

\section{INTRODUCTION}

Virtual reality (VR) technology enables the depiction of physical reality in an artificial environment. Recent developments in game engines and the increase in the number of free software such as Unity, Unreal Engine, CryEngine, Armory have surged the interest in VR technologies. With rising interest, VR systems began to be employed in various scientific and commercial applications. VR technology has proven itself to be a powerful tool for the visualization of geospatial data and generating 3D photo-realistic models from imagery in the applications such as virtual touring, cultural heritage visualization, forensic examination, and maritime archaeology (Koutsoudis et al., 2007; Putra et al., 2016; Doležal et al., 2019; Koller et al., 2019). Nowadays, the ongoing Covid19 pandemic and thus the requirement to stay at home as a safety precaution against the risk of infection has limited people's mobility. Due to this fact, many organizations changed their structure and provided opportunities to work from home to the employees. Because of these restrictions, virtual tours that enable remote visits, have become popular and this situation has led to the expansion of VR applications. Some studies even suggest that VR tourism during the pandemic has become a source of revenue for various attractions and these remote visits also have a positive impact on the physiological well-being of the visitors (Itani and Hollebeek, 2021; Li et al., 2021). VR tours can be in different forms such as $360^{\circ}$ virtual tours that are produced through the combination of panoramic images taken in different locations, and 3D virtual tours which are created by the integration of high-quality 3D models into an artificial environment.

Photogrammetry is a branch of remote sensing, that extracts information from aerial photos based on mathematical equations and models. In cultural heritage documentation, archaeological research, architectural applications, 3D modelling of objects such as buildings, bridges, trees, and even dinosaur footprints can be carried out by employing photogrammetric techniques (Guarnieri et al., 2006; Shashi and Jain., 2007; Petti et al., 2008; Riveiro et al., 2011; Miller et al., 2015). With the appearance of unmanned air vehicles (UAVs) which are remotely or manually controlled unmanned flying platforms, a new source as an alternative to aircraft has emerged in photogrammetric studies. With rapid technological development, UAV has become an indispensable technique for high-quality $3 \mathrm{D}$ modelling of the terrain and non-terrain objects by means of offering very high spatial resolution, low cost, and fast data processing time. In addition, UAV data is used in varied applications such as cultural heritage documentation, archaeological surveys, inspections of engineering constructions and deformation analysis of natural disasters (Eisenbeiss and Zhang, 2006; Fernández-Hernandez et al., 2015; Yamazaki et al., 2015; Khaloo et al., 2018). UAV imagery is generally processed in structure from motion (SfM) based photogrammetric evaluation software such as Agisoft Metashape, Pix4D, Visual SFM and Context Capture (Lucieer et al., 2014; Nesbit and Hugenholtz, 2019; Sefercik et al., 2019). In these software, 3D model generation is completed by using a dense point cloud, produced after aligning aerial photos based on the SFM algorithm.

In this study, it is aimed to create a 3D virtual tour for the Gebze Technical University (GTU) Southern Campus by integrating high-resolution UAV data, processed in SfM based photogrammetric evaluation software Agisoft Metashape, into a virtual environment. With the creation of the 3D VR tour, candidate students, academicians, other visitors will be able to visit the Campus remotely in a high-resolution artificial environment and get information about the terrain and nonterrain objects. At the same time, the VR tour will provide convenience to visitors during the ongoing pandemic period. 
According to the aims, the study consists of five sections. In the next section, information about the study area and the properties of used materials are given. The third section presents the used methodology in 3D textured mesh model generation and the creation of a 3D virtual Campus tour. The results of the study are shown in the fourth section, followed by the conclusion.

\section{STUDY AREA AND MATERIALS}

The study area is the Southern Campus of GTU, located at Gebze district of Kocaeli province. The Campus covers $0.5 \mathrm{~km}^{2}$ between $40^{\circ} 48^{\prime} 21^{\prime \prime}$ East longitude and 29 21' 33" North parallel. Different land cover classes such as buildings, roads, and vegetation exist in the area and the topography is generally flat. Figure 1 presents the location of the study area.

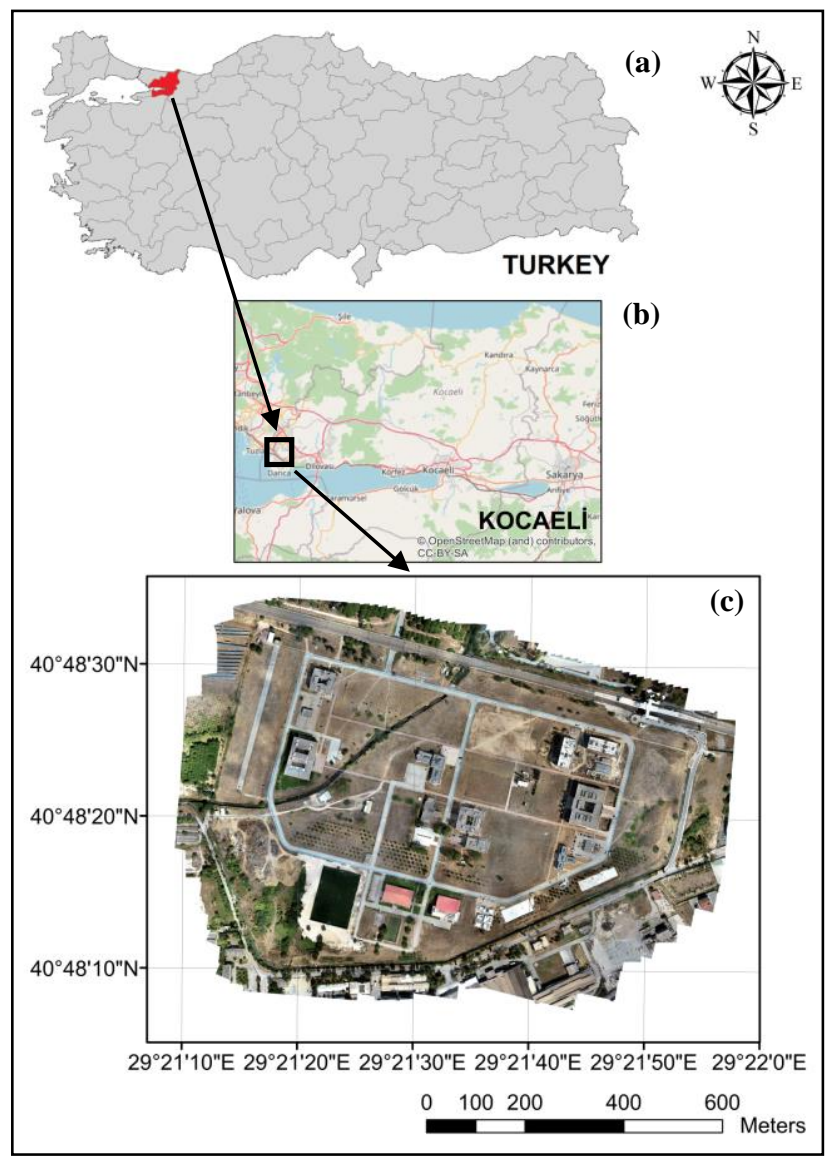

Figure 1. Position of the Kocaeli province in Turkey (a), location of GTU in Kocaeli (b) and orthomosaic map of GTU Southern Campus in geographic coordinate system and WGS84 datum (c)

In UAV flights and GNSS (Global Navigation Satellite System) surveys, DJI Phantom IV Pro V2.0 UAV and CHC i80 GNSS receiver, available at GTU Geomatics Engineering Department's Advanced Remote Sensing Technology Laboratory, were used. In Table 1, used equipment and their specifications are shown.

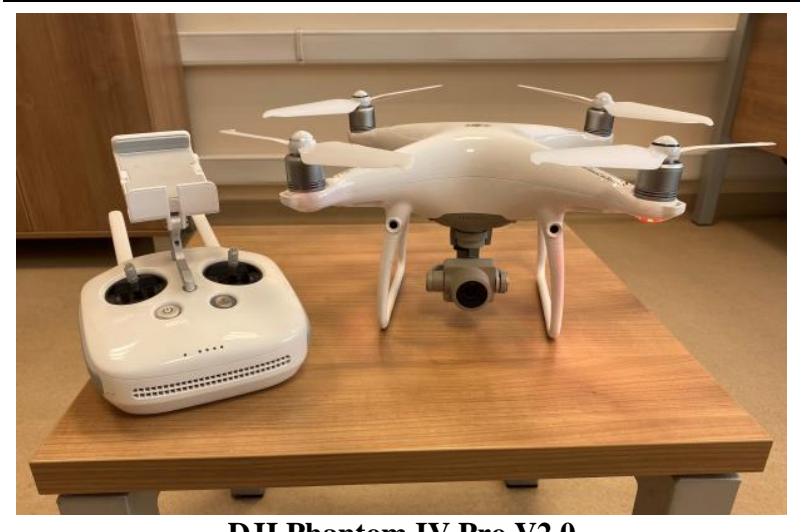

DJI Phantom IV Pro V2.0

\begin{tabular}{ll}
\hline \multicolumn{1}{c}{ Specification } & \multicolumn{1}{c}{ Value } \\
\hline Camera & $\begin{array}{l}\text { 4K, HD, 1080p, 1”, effective } \\
\text { pixels 20 MP }\end{array}$ \\
\hline Gimbal & 3-axis (pitch, roll, yaw) \\
\hline Flight duration & Max. 30 minutes \\
\hline Weight & $1375 \mathrm{~g}$ \\
\hline Speed & Max $20 \mathrm{~m} / \mathrm{s}$ in S-mode \\
\hline Wind speed resistance & Max. 10 m/s \\
\hline Operating temperature & $0^{\circ}$ to $40^{\circ} \mathrm{C}$ \\
\hline $\begin{array}{l}\text { Outdoor positioning } \\
\text { module }\end{array}$ & GPS/GLONASS dual \\
\hline Hover Accuracy Range & $\begin{array}{l} \pm 0.1 \mathrm{~m} \mathrm{~V}, \pm 0.5 \mathrm{~m} \mathrm{H} \text { (Vision); } \\
\pm 0.3 \mathrm{~m} \mathrm{~V}, \pm 1.5 \mathrm{~m} \mathrm{H} \text { (GPS) }\end{array}$
\end{tabular}

$\begin{array}{ll} & \\ & \end{array}$

Table 1. Specifications of used UAV and GNSS receiver 


\section{METHODOLOGY}

The applied methodologies were presented in two flow charts, shown in Figure 2a and 2b. Where, Figure 2a includes the processing steps for 3D textured mesh model generation, Figure $2 \mathrm{~b}$ comprises the used steps in the integration of generated $3 \mathrm{D}$ mesh models into the Unity game engine for creating a 3D VR tour application. The matching and geometric correction of aerial photos, dense cloud generation and filtering, and 3D textured mesh model production were completed in Agisoft Metashape software. For importing produced 3D textured mesh models into the virtual domain and designing a VR tour application, the Unity game engine was utilized.

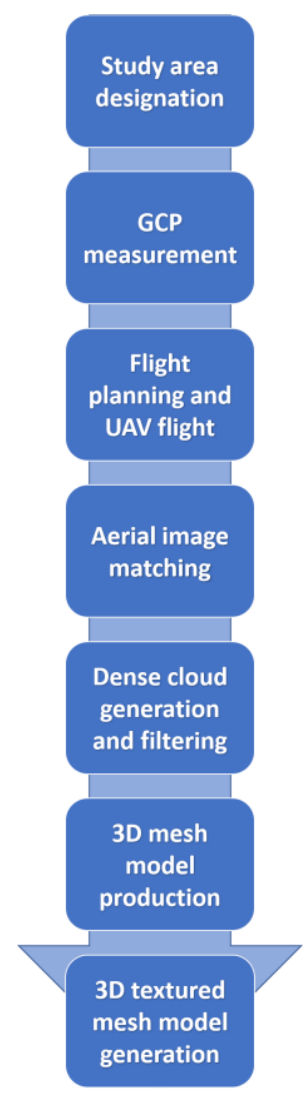

a

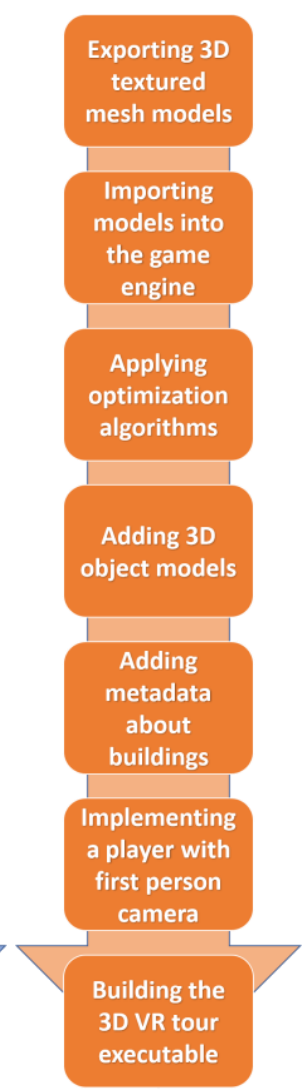

b
Figure 2. Used methodologies of the study; 3D textured mesh model generation steps (a), 3D VR tour creation steps (b)

\subsection{GCP Measurement and Flight Planning}

For the orientation of aerial images, 43 ground control points (GCPs) were established over the study area. The established GCPs were measured by Continuously Operating Reference Stations (CORS) Real Time Kinematic (RTK) GNSS technique with $0.8 \mathrm{~cm}$ horizontal and $1.5 \mathrm{~cm}$ vertical relative positioning accuracy. In flight planning, the main aim was to cover the whole $3 \mathrm{D}$ modelling area with a minimum number of photos without any remarkable gaps. Therefore, using Pix4Dcapture UAV flight planning software, the entire area was covered by bundle grid, polygonal and circular flights. Bundle grid and circular flights were applied to the buildings for more realistic 3D building models. Where $80 \mathrm{~m}$ altitude was preferred for the bundle grid and polygonal flights, circular flights were completed with an altitude of $30 \mathrm{~m}$ to achieve the GSD of $\leq 2.18$ $\mathrm{cm}$. In prepared flight plans, the minimum front and side overlap ratios were $80 \%$ and $60 \%$ respectively. Finally, a total of 5161 aerial photos were acquired with the flights. In Figure 3 , the prepared flight plan over the study area is shown. According to the flight plan, four bundle grid and three polygonal flights were realized.

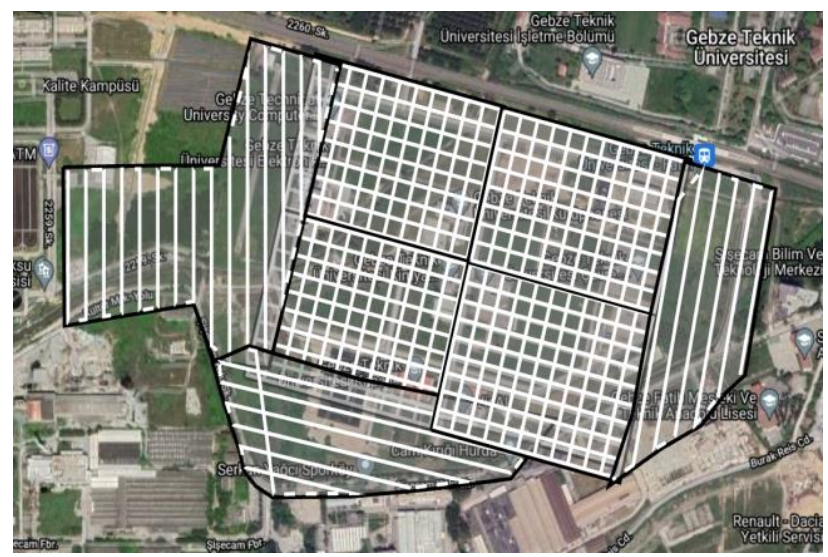

Figure 3. Prepared flight plan

\subsection{Matching of Aerial Photos}

As mentioned before, matching of aerial photos was carried out using a SfM based software Agisoft Metashape Professional on the high-end HP Z4 G4 workstation which is equipped with Intel ${ }^{\circledR}$ Xeon ${ }^{\circledR}$ W-2133 CPU @ $3.60 \mathrm{GHz}, 64$ GB RAM and an NVIDIA Quadro P2000 graphics card. The SfM algorithm is a low-cost and efficient photogrammetric technique that allows the generation of 3D structures in high resolution by utilizing an array of offset photos with certain overlap ratios (Westoby et al., 2012). As shown in Figure 4, the SfM technique requires a series of offset photos with overlap to extract features and reconstruct 3D geometry.

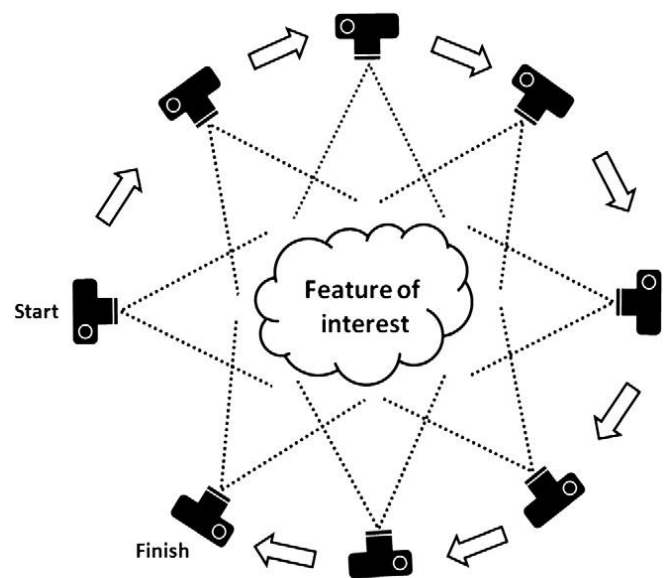

Figure 4. Principle of photo acquisition geometry in the SfM algorithm (Westoby et al., 2012)

The matching procedure consists of two main steps as the initial image alignment to generate a sparse point cloud as the primary model and geometric correction. Before the initial image alignment, obtained aerial photos were imported into Agisoft Metashape software as five different tiles for a shorter processing time due to high CPU and RAM capacity requirements for more than 2000 aerial photos (threshold number of photos for SfM-based image matching software). Camera calibration was carried out automatically for the determination of interior orientation parameters. Also, the 
images with the background information, captured with offnadir orientation, have been masked manually to eliminate the disruptive effect of background pixels on the image alignment. After the camera calibration was performed and background pixels were masked, initial image alignment was realized for the purpose of generating the primary model, a sparsely populated point cloud with more than 48 million points. The geometry of the produced model was corrected by marking GCPs, measured before the UAV flights. The GCPs were imported as a reference into the Agisoft Metashape and marked in aerial photos (Figure $5)$.

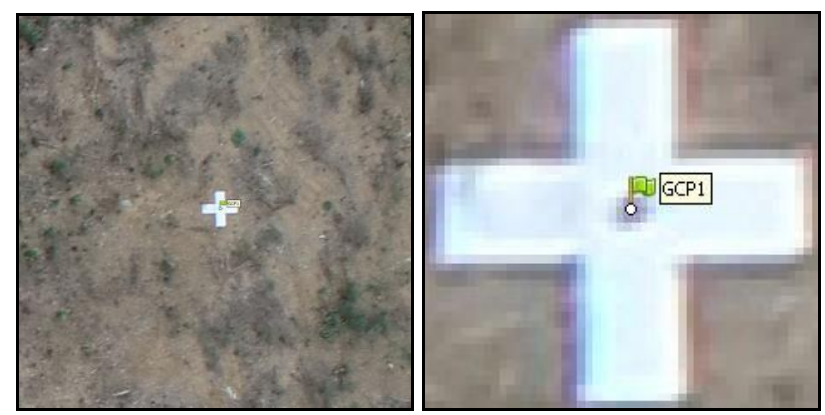

Figure 5. A sample GCP on an aerial photo

The geometric accuracy based on marked GCPs was automatically calculated as the root mean square error (RMSE) in both meters and pixels according to Equation (1). Finally, geometric accuracy was calculated as $\pm 8 \mathrm{~mm}$ ( $\sim 0.4$ pixels).

$$
\text { RMSE }=\sqrt{\frac{\sum_{\mathrm{i}=1}^{n}\left(\hat{X}_{\mathrm{i}}-X_{\mathrm{i}}\right)^{2}+\left(\hat{Y}_{\mathrm{i}}-Y_{\mathrm{i}}\right)^{2}+\left(\hat{Z}_{\mathrm{i}}-Z_{\mathrm{i}}\right)^{2}}{n}}
$$

where $\quad \hat{X}_{i}, \hat{Y}_{i}, \hat{Z}_{i}=$ estimated values for i camera position $\mathrm{X}_{\mathrm{i}}, \mathrm{Y}_{\mathrm{i}}, \mathrm{Z}_{\mathrm{i}}=$ input values for $\mathrm{i}$ camera position

\subsection{Dense Cloud Generation and Filtering}

To produce a high-quality 3D mesh model, a high-resolution densely populated point cloud consisting of approx. one billion points were generated by processing depth maps, built by extracting depth information from the image pairs with high overlapping values. The dense cloud generation with more than 5000 aerial photos took 5-12 hours per tile even using a computer with a RAM capacity of 64 GB. Generated dense point clouds have noise because of distortions in imaging geometry and the influence of land cover characteristics (especially forest). Of course, the noise may affect the quality of the generated 3D models. In this study, the noisy parts of the point clouds were classified and filtered. In addition, various objects such as cars, benches, lighting poles, and traffic signs were filtered as they might cause noise. Figure 6 shows the dense point cloud before and after the filtering process.

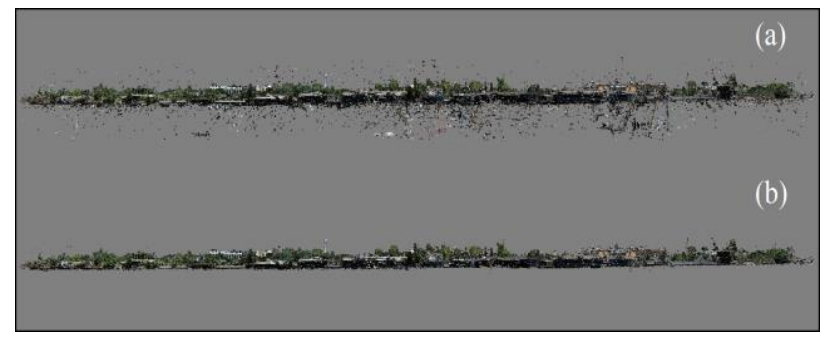

Figure 6. Dense cloud with noise data (a) and filtered dense cloud (b)

\subsection{D Textured Mesh Model Generation}

Generated dense point cloud determines the objects in the noncontinuous point-based format, so for proper visualization and thus creating an immersive VR tour it was essential to reconstruct objects in 3D solid form. Therefore, processing dense point cloud data, 3D mesh models were produced. Due to the sheer number of predicted faces (triangle) in the 3D mesh model preconstruction phase, the mesh decimation process was applied and the total number of faces was decreased to approx. 70 million. 3D textured mesh model was generated by employing aerial photos to produce high-resolution textures and then applying these textures to 3D mesh models. The generated 3D textured mesh models were filtered because of containing isolated polygons and objects with rough geometry.

\subsection{D VR Tour Creation}

Integration of the 3D mesh models into a virtual domain is essential in creating a 3D VR tour application. Efficient integration of $3 \mathrm{D}$ mesh models requires exporting them in a suitable format. Therefore, generated 3D textured mesh models were exported in Wavefront OBJ format and imported into the Unity game engine which supports OBJ files. Wavefront OBJ format stores coordinates of points, polygonal data of 3D models, and it is commonly employed and supported by an array of 3D computer graphics software (Kato and Ohno, 2009). Because of the large number of faces in imported 3D models and thus large polygonal data, rendering optimization algorithms such as occlusion culling, and space subdivision were applied for increasing performance and efficiency. Occlusion culling algorithms are applied to identify, render visible sections of the 3D models, thus objects that fall behind the visible parts of a particular point of view are hidden and not rendered to increase the performance, eliminate hardware bottlenecks (Coorg and Teller, 1997). Both occlusion culling and space subdivision do not have a considerable effect on processing time. To improve the visualization, 3D object models such as trees, lighting poles and arbours were positioned on the VR application. Furthermore, textual metadata about buildings such as name, total floor area, and total usage area was added to inform the visitors about the Campus. To construct an immersive VR tour experience, users should be able to walk around buildings and objects as if they are walking in real life. Therefore, a player with a first-person camera was added for a realistic virtual experience. Finally, an executable file was built in Unity for the 3D VR tour application.

\section{RESULTS}

Figures 7 and 8 show the generated sparse point cloud and dense point cloud respectively.

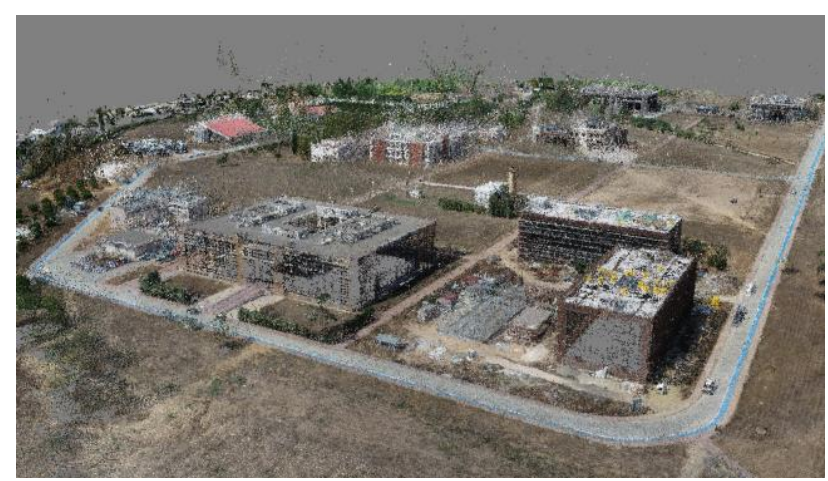

Figure 7. Generated sparse point cloud 


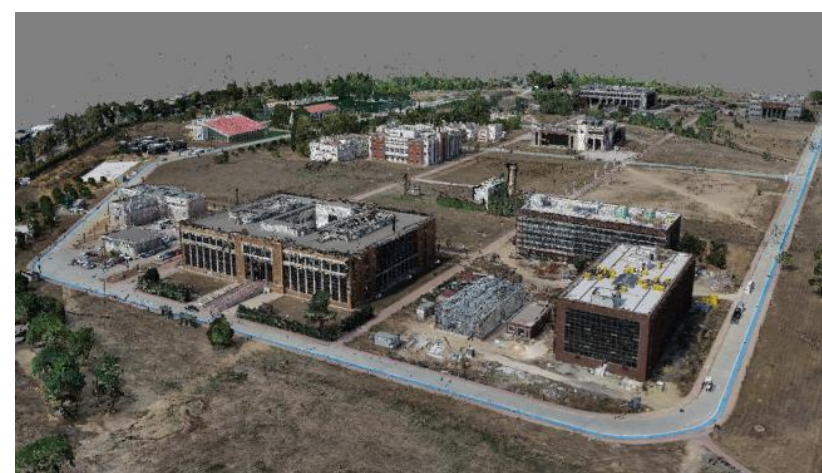

Figure 8. Produced dense point cloud

The point clouds were generated by merging five studied tiles. The dense point clouds of the independent tiles were generated in high resolution and the description potentials of the clouds were sufficient even in building corners. Figure 9 illustrates an instance of generated 3D textured mesh models.

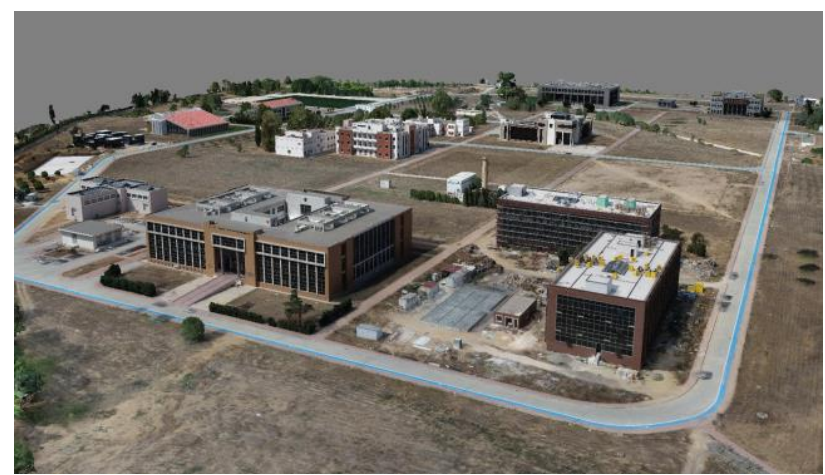

Figure 9. 3D textured mesh model of the Campus

3D textured mesh models were produced in high quality however small distortions exist due to the interpolation effects on particularly non-continuous vertical objects such as rooftop walls and eaves. Figure 10 shows the development of the 3D VR tour application in the Unity game engine.

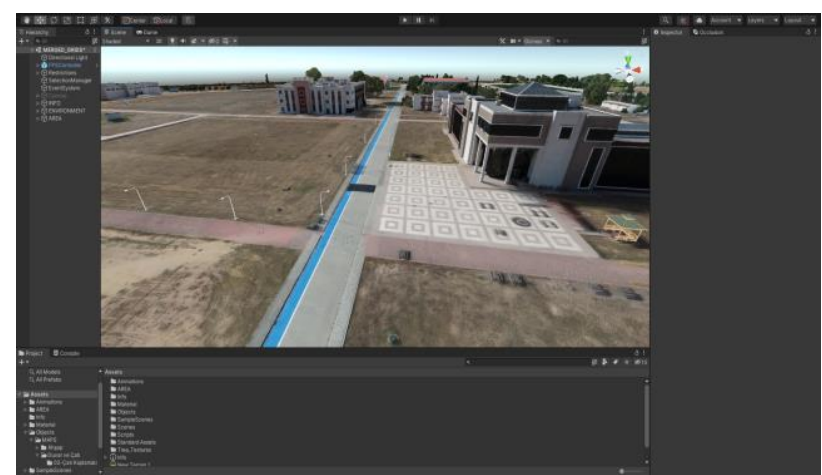

Figure 10. Development of 3D VR tour application in Unity

Figure $11 \mathrm{a}$ and $11 \mathrm{~b}$ display samples from the first-person point of view of the created 3D VR tour application. By means of very high-resolution UAV data, the contents of the Campus were introduced in detail successfully.
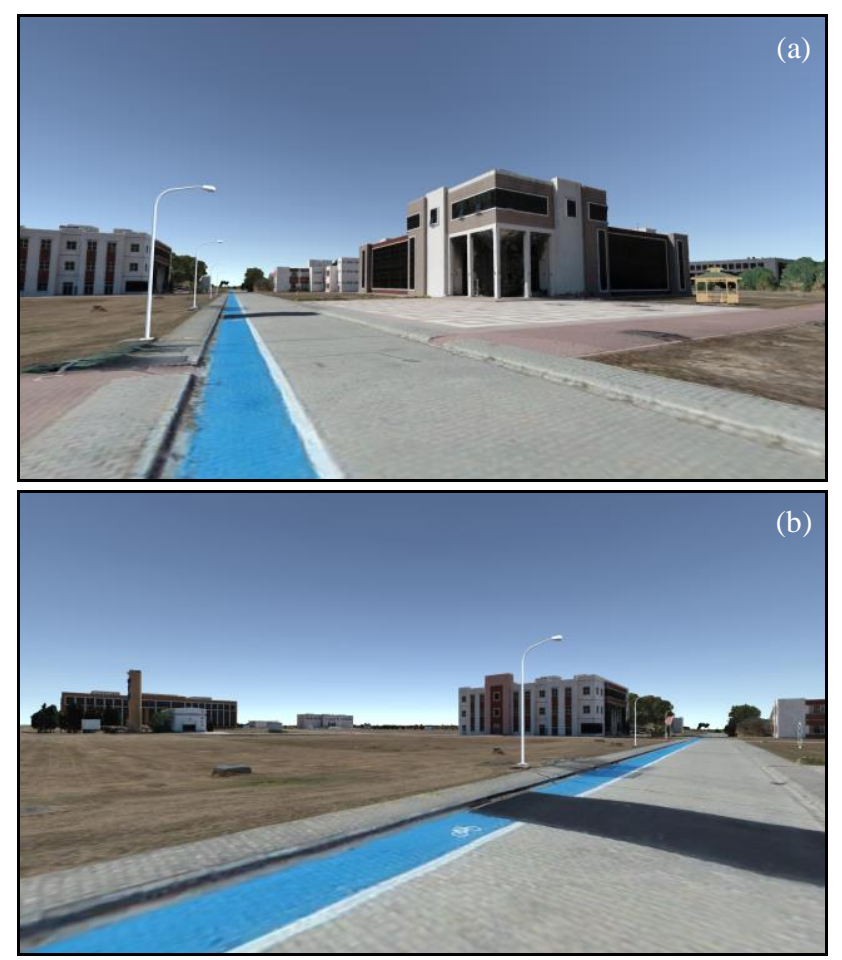

Figure 11. Some sample scenes from the 3D VR tour application in first-person point of view (a), (b)

In VR application figures, premade 3D object models, placed for better visualization, can be seen. In addition, interactive information panels with the pop-up window feature were located near buildings to display textual metadata (Figure 12).

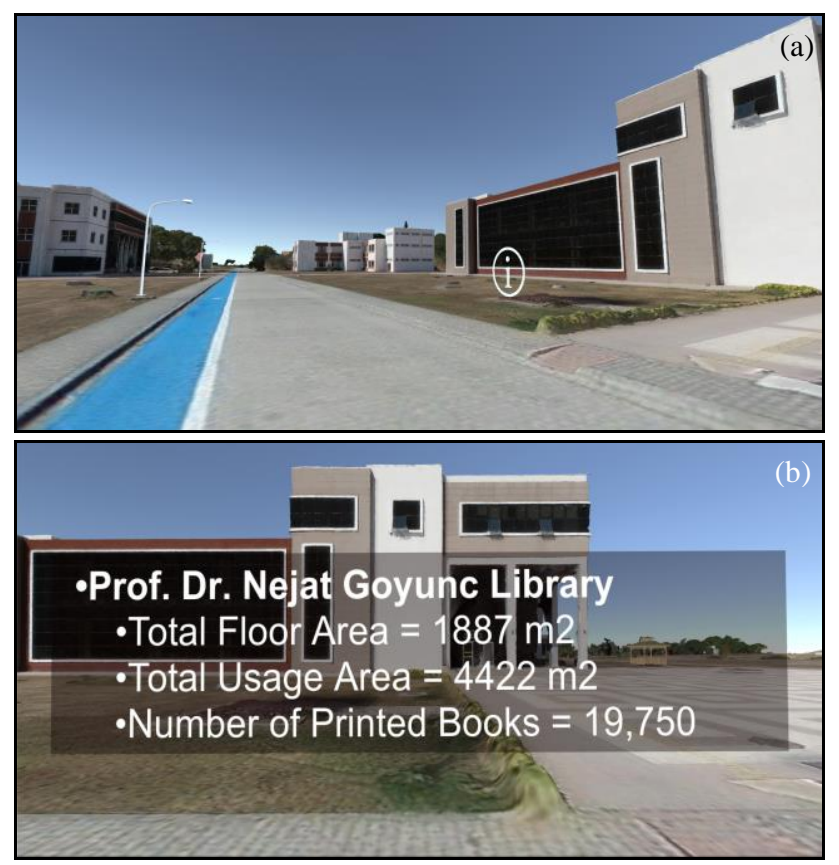

Figure 12. Information panel symbol in the $3 D$ VR tour application (a) and the pop-up window showing the textual metadata about the building (b)

The achieved results demonstrated that the 3D VR tour application offers users the opportunity to explore realistic 3D 
models, collect information about Campus contents remotely, and have an immersive virtual experience.

\section{CONCLUSIONS}

With the recent developments in game engines, VR applications gained popularity and the adoption of UAV data for displaying high resolution geospatial data in a virtual environment became a significant topic. In this study, the potential of UAV imagery in generating high-quality $3 \mathrm{D}$ textured mesh models and integrating these models into a virtual domain for creating a 3D VR tour application was tested. High-resolution aerial photos of the GTU Southern Campus were captured by using a UAV with 20 megapixel RGB camera, in different capturing geometries such as bundle grid, polygonal, and circular. Acquired UAV photos were oriented in SfM-based image matching software Agisoft Metashape and using 43 independent GCPs, the geometry was corrected with RMSE of $\pm 8 \mathrm{~mm}(\sim 0.4$ pixels). After these processes, dense point clouds were generated, and employing these dense clouds high-quality 3D textured mesh models were produced. Integration of produced $3 \mathrm{D}$ textured mesh models into a virtual domain was carried out by importing $3 \mathrm{D}$ models into the Unity game engine. Then the 3D object models such as lighting poles, trees, arbours, benches were placed to improve the visualization. Also, the textual metadata about buildings such as name, total floor area, total usage area was added to inform users about the Campus contents during the VR tour. Finally, a player with a first-person camera was integrated for a realistic VR experience. Overall, thanks to the high-resolution UAV imagery, high-quality 3D textured models were produced and successfully integrated into the virtual domain using a game engine for the creation of an immersive 3D VR tour application. The generated tour will be served through the GTU official website as a web-based application and a downloadable file.

\section{ACKNOWLEDGEMENTS}

We would like to thank Gebze Technical University for supporting this research in the scope of a scientific research project (ID: 2020-A-105-42).

\section{REFERENCES}

Coorg, S., Teller, S., 1997. Real-time occlusion culling for models with large occluders. In Proceedings of the Symposium on Interactive 3D Graphics, USA, 83-90.

Doležal, M., Vlachos, M., Secci, M., Demesticha, S., Skarlatos, D., Liarokapis, F., 2019. Understanding Underwater Photogrammetry for Maritime Archaeology Through Immersive Virtual Reality. Int. Arch. Photogramm. Remote Sens. Spatial Inf. Sci., XLII-2/W10, 85-91. https://doi.org/10.5194/isprsarchives-XLII-2-W10-85-2019

Eisenbeiss, H., Zhang, L., 2006. Comparison of DSMs generated from mini UAV imagery and terrestrial laser scanner in a cultural heritage application. Int. Arch. Photogramm. Remote Sens. Spatial Inf. Sci., 36(5), 90-96.

Fernández-Hernandez, J., González-Aguilera, D., Rodríguez-Gonzálvez, P., Mancera-Taboada, J., 2015. Image-based modelling from unmanned aerial vehicle (UAV) photogrammetry: an effective, low-cost tool for archaeological applications. Archaeometry, 57(1), 128-145. https://doi.org/10.1111/arcm.12078
Guarnieri, A., Remondino, F., Vettore, A., 2006. Digital photogrammetry and TLS data fusion applied to Cultural Heritage 3D modeling. Int. Arch. Photogramm. Remote Sens. Spatial Inf. Sci., 36(5), 1-6.

Itani, O. S., Hollebeek, L. D., 2021. Light at the end of the tunnel: Visitors' virtual reality (versus in-person) attraction site tour-related behavioral intentions during and post-COVID-19. $\begin{array}{llll}\text { Tourism Management, } & 84, & 104290 .\end{array}$ https://doi.org/10.1016/j.tourman.2021.104290

Kato, A., Ohno, N., 2009. Construction of three-dimensional tooth model by micro-computed tomography and application for data sharing. Clinical Oral Investigations, 13(1), 43-46. https://doi.org/10.1007/s00784-008-0198-4

Khaloo, A., Lattanzi, D., Cunningham, K., Dell'Andrea, R., Riley, M., 2018. Unmanned aerial vehicle inspection of the Placer River Trail Bridge through image-based 3D modelling. Structure and Infrastructure Engineering, 14(1), 124-136. https://doi.org/10.1080/15732479.2017.1330891

Koller, S., Ebert, L. C., Martinez, R. M., Sieberth, T., 2019. Using virtual reality for forensic examinations of injuries. Forensic Science International, 295, 30-35. https://doi.org/10.1016/j.forsciint.2018.11.006

Koutsoudis, A., Arnaoutoglou, F., Chamzas, C., 2007. On 3D reconstruction of the old city of Xanthi. A minimum budget approach to virtual touring based on photogrammetry. Journal of Cultural Heritage, 8(1), 26-31. https://doi.org/10.1016/j.culher.2006.08.003

Li, Y., Song, H., Guo, R., 2021. A Study on the Causal Process of Virtual Reality Tourism and Its Attributes in Terms of Their Effects on Subjective Well-Being during COVID-19. International Journal of Environmental Research and Public Health, 18(3), 1019. https://doi.org/10.3390/ijerph18031019

Lucieer, A., Jong, S. M. D., Turner, D., 2014. Mapping landslide displacements using Structure from Motion (SfM) and image correlation of multi-temporal UAV photography. Progress in Physical Geography, 38(1), 97-116. https://doi.org/10.1177/0309133313515293

Miller, J., Morgenroth, J., Gomez, C., 2015. 3D modelling of individual trees using a handheld camera: Accuracy of height, diameter and volume estimates. Urban Forestry \& Urban Greening, 14(4), 932-940. https://doi.org/10.1016/j.ufug.2015.09.001

Nesbit, P. R., Hugenholtz, C. H., 2019. Enhancing UAV-SFM 3D model accuracy in high-relief landscapes by incorporating oblique images. Remote Sensing, 11(3), 239. https://doi.org/10.3390/rs11030239

Petti, F. M., Avanzini, M., Belvedere, M., De Gasperi, M., Ferretti, P., Girardi, S., Tomasoni, R., 2008. Digital 3D modelling of dinosaur footprints by photogrammetry and laser scanning techniques: integrated approach at the Coste dell'Anglone tracksite (Lower Jurassic, Southern Alps, Northern Italy). Acta Geologica, 83, 303-315.

Putra, E. Y., Wahyudi, A. K., Dumingan, C., 2016. A proposed combination of photogrammetry, Augmented Reality and Virtual Reality Headset for heritage visualisation. In IEEE 
International Conference on Informatics and Computing, Indonesia, 43-48. https://doi.org/10.1109/IAC.2016.7905687

Riveiro, B., Caamaño, J. C., Arias, P., Sanz, E., 2011. Photogrammetric 3D modelling and mechanical analysis of masonry arches: An approach based on a discontinuous model of voussoirs. Automation in Construction, 20(4), 380-388. https://doi.org/10.1016/j.autcon.2010.11.008

Sefercik, U. G., Tanrikulu, F., Atalay, C., 2019. Photogrammetric 3D modelling potential comparison of SFMbased new generation image matching software. In the 40th Asian Conference on Remote Sensing, Korea, 1-8.

Shashi, M., Jain, K., 2007. Use of photogrammetry in 3D modeling and visualization of buildings. Journal of Engineering and Applied Sciences, 2(2), 37-40.

Westoby, M. J., Brasington, J., Glasser, N. F., Hambrey, M. J., Reynolds, J. M., $2012 . \quad$ 'Structure-fromMotion'photogrammetry: A low-cost, effective tool for geoscience applications. Geomorphology, 179, 300-314. https://doi.org/10.1016/j.geomorph.2012.08.021

Yamazaki, F., Matsuda, T., Denda, S., Liu, W., 2015. Construction of 3D models of buildings damaged by earthquakes using UAV aerial images. In Proceedings of the 10th Pacific Conference on Earthquake Engineering Building an Earthquake-Resilient Pacific, Australia, 1-8, 204. 